Avaliação da rotulagem e fracionamento de produtos cárneos comercializados na cidade de Bom Jesus-PI

\title{
Evaluation of the labeling and fractionation of meat products commercialized in the city of Bom Jesus-PI
}

\section{Paulo Gomes do Nascimento Corrêa1, Wenderson Rodrigues de Amorim², Eveny Silva de Melo² $^{2}$ Raizza Eveline Escórcio Pinheiro ${ }^{3}$}

\begin{abstract}
Resumo: As coletas de dados foram realizadas nas três maiores redes de supermercados da região, que comercializam seus produtos na forma de atacado. As visitas ocorreram no segundo semestre de 2016 e primeiro semestre de 2017, sempre na primeira semana de cada mês, sendo uma loja por dia, em qualquer dos três períodos, com cronograma e ordem escolhido aleatoriamente através de sorteio. Em cada visita a coleta de dados foi realizada através da aplicação de checklist. Cada produto teve registro fotográfico caracterizando as condições de venda, identificação da rotulagem em geral e a informação nutricional. Os produtos cárneos selecionados foram: linguiça, salame, presunto, salsicha, jerked beef e charque. Foi realizada ainda a avaliação das não conformidades quanto ao fracionamento realizado pelos estabelecimentos que comercializam os produtos citados. Após análise dos resultados, concluiu-se que os supermercados avaliados apresentaram pouca variação na distribuição das marcas dos produtos analisados. O percentual de não conformidades foi maior nos produtos fracionados, em comparação aos produtos originais. Os produtos fracionados não exibem todas as informações necessárias à compreensão dos consumidores, impedindo dessa forma, a rastreabilidade do produto.
\end{abstract}

Palavras-chave: checklist, fracionados, não conformidades, produtos de origem animal.

Abstract: This study was developed in the city of Bom Jesus-PI, with the objective of evaluating the labeling and fractionation of selected meat products from establishments with SIF. The data was collected in the three largest supermarket chains in the region, which sell their products in the form of wholesale. Visits occurred in the second half of 2016 and the first half of 2017, always in the first week of each month, one store per day, in any of the three periods, with a schedule and order chosen randomly. At each visit the data collection was performed through the application of checklist. Each product had a photographic record characterizing the conditions of sale, identification of the labeling and the nutritional information. The meat products selected were: sausage, salami, ham, sausage, jerked beef and charqui meat. The evaluation of the nonconformities regarding the fractionation carried out by the establishments that market the cited products was also carried out. After analyzing the results, it was concluded that the supermarkets evaluated showed little variation in the distribution of the brands of the analyzed products. The percentage of nonconformities was higher in fractionated products, compared to the original products. Fractionated products do not display all the information necessary for consumer understanding, thus preventing product traceability.

Keywords: checklist, fractionated, nonconformities, products of animal origin.

Autor para correspondência. E-mail: paulonascimento.pi@gmail.com

Recebido em 10.2.2019. Aceito em 31.3.2019

http://dx.doi.org/10.5935/1981-2965.20190009

${ }^{1}$ Discente de Medicina Veterinária - Universidade Federal do Piauí, Campus Professora Cinobelina Elvas, Bom Jesus, Piauí, Brasil. E-mail: paulonascimento.pi@gmail.com 
${ }^{2}$ Graduado em Medicina Veterinária pela Universidade Federal do Piauí, Bom Jesus - PI, Brasil. E-mail: rodriguesamorim10@gmail.com, evenymelo06@ hotmail.com

3Professora Doutora - Universidade Federal do Piauí, Teresina, Piauí, Brasil. E-mail: raizza_eveline@hotmail.com

\section{Introdução}

É de extrema importância a existência de legislações que regulamentam as normas de rotulagem de produtos alimentícios. Na elaboração de um rótulo do alimento devem constar todas as informações fundamentais para que o consumidor saiba o que está adquirindo, assim como fornecer todas as bases necessárias para que os órgãos competentes realizem seu trabalho de fiscalização (ALMEIDA-MURADIAN; PENTEADO; Ano.. GONÇALVES, 2010).

Diversos fatores podem influenciar na decisão de compra dos alimentos pelos consumidores. Estes por sinal consideram o preço, qualidade e atributos sensoriais, como sabor e cor, mas também podem considerar se o alimento foi produzido utilizando novas tecnologias. Atualmente, o rótulo alimentício vem sendo estudado principalmente como fonte de informação nutricional aos consumidores, influenciando em suas escolhas alimentares.

A rotulagem dos alimentos embalados é obrigatória e está regulamentada pela legislação brasileira através de órgãos como o Ministério da
Saúde por meio da Agência Nacional de Vigilância Sanitária (ANVISA), o Ministério da Agricultura, Pecuária e Abastecimento (MAPA) e o Instituto Nacional de Metrologia, Qualidade e Tecnologia (INMETRO) (MACHADO, 2015). Os produtos de origem animal (POA) que atendem as normas técnicas que permitem o comércio internacional, padronizadas pelo Codex Alimentarius (CODEX ALIMENTARIUS, 2010), devem possuir no Brasil um Regulamento Técnico de Identidade e Qualidade (RTIQ) emitido pelo MAPA que possui diversos parâmetros de exigências, a partir dos processos de elaboração, rotulagem, até a composição química que deverão ser atendidas pelo fabricante do produto (BRASIL, 2001). Recentemente, o MAPA lançou o Decreto $\mathrm{n}^{\mathrm{o}}$ 9.013, onde a rotulagem de produtos de origem animal passou a ser automática e instantânea, diminuindo assim o tempo de registro dos produtos (BRASIL, 2017).

De modo geral os produtos que possuem RTIQ são aqueles de maior consumo no Brasil e em consequência de maior produção como, por exemplo, linguiça e salsicha (BRASIL, 2000a), bacon (BRASIL, 2000b), jerked beef 
(BRASIL, 2000c), carne bovina moída (BRASIL, 2003), entre outros quais. Conforme o Regulamento da Inspeção Industrial e Sanitária de Produtos de Origem Animal (RIISPOA), todos os produtos de origem animal entregues ao comércio devem estar identificados por meio de rótulos registrados, além de serem acondicionados ou embalados em recipientes ou continentes que confiram a necessária proteção, atendidas as características específicas do produto e as condições de armazenamento e transporte (BRASIL, 2017).

No entanto, muitas vezes na prática, detecta-se que as legislações e as fiscalizações não são suficientes para coibir a existência de não conformidades associadas a fraudes, informações insuficientes e/ou inadequadas, prejudicando assim o consumidor. Dessa forma, considerando a importância da inspeção dos POA e da rotulagem dos alimentos em geral, esta pesquisa foi realizada com o objetivo de avaliar a rotulagem e o fracionamento dos produtos cárneos selecionados procedentes de estabelecimentos com SIF, comercializados em Bom Jesus, PI.

\section{Material e Métodos}

\subsection{Local}

O presente trabalho foi realizado na cidade de Bom Jesus-PI, com o objetivo de avaliar a rotulagem e o fracionamento de produtos cárneos selecionados procedentes de estabelecimentos com SIF.

Foram escolhidas estrategicamente de forma aleatória três lojas que representam as maiores redes de supermercado. Os três estabelecimentos que comercializam seus produtos na forma de atacado, foram denominados de "A", "B" e "C". Fizeram parte da pesquisa todas as variedades de marcas existentes em cada estabelecimento dos produtos selecionados. Por questões éticas e com intuito de preservar a identidade dos locais de pesquisa, e das marcas comerciais, estas não foram identificadas.

As amostras para o desenvolvimento do presente estudo foram constituídas de POA selecionados, que possuem RTIQ ou qualquer padronização oficial do MAPA, provenientes de qualquer região do país, comercializados no segundo semestre de 2016 e primeiro semestre de 2017. Os produtos cárneos selecionados foram: linguiça, salame, presunto, salsicha, jerked beef e charque. Foi realizada ainda a avaliação das não conformidades quanto ao fracionamento realizado pelos estabelecimentos que comercializam os produtos citados.

A frequência das visitas aos supermercados era realizada na primeira semana de cada mês, sendo uma loja por 
dia, em qualquer dos três períodos, com cronograma e ordem escolhido aleatoriamente através de sorteio. Os parâmetros analisados dos produtos cárneos selecionados foram: identificação do produto e avaliação das condições de comercialização; análise da relação de ingredientes; análise da informação nutricional; análise da rotulagem e análise do fracionamento.

A coleta dos dados individuais foi efetuada por aplicação de checklist. Para elaboração do checklist foram seguidas as recomendações determinadas pelo Código de Defesa do Consumidor (BRASIL, 1990), pela Resolução RDC $\mathrm{n}^{\circ} 13$ (BRASIL, 2001), pela Instrução Normativa n 22 (BRASIL, 2005) e pela Resolução RDC no 360 (BRASIL, 2003).

Para cada produto também era realizado um registro fotográfico caracterizando as condições de venda, identificação da rotulagem em geral e a informação nutricional. A avaliação das condições de comercialização ficou restrita aos produtos selecionados e foi realizada no momento de sua identificação. Foram avaliadas ainda, a integridade da embalagem e da rotulagem e as recomendações do fabricante quanto a temperatura e prazo de validade.

\section{Análises estatísticas}

Os resultados foram analisados e descritos em relação às conformidades $(\mathrm{C})$ e não conformidades (NC) verificadas. Em seguida, os mesmos foram tabulados e transformados em percentual com auxílio do Programa Microsoft Office Excel 2010.

\section{Resultados e discussão}

A Tabela 1 apresenta os resultados das conformidades e não conformidades encontradas após aplicação do checklist para linguiças. Observou-se ausência de inconformidades entre os estabelecimentos A, B e C para as marcas I e II. De todos os itens avaliados, a marca III foi a única que apresentou inconformidades nos três locais de pesquisa. Dentre as não conformidades encontradas na rotulagem de linguiças da marca III nos estabelecimentos A e B destaca-se principalmente: a ausência de lote; informações obrigatórias como datas de fabricação e validade ausentes; como também omitiam informações do teor de gorduras trans.

Já para o estabelecimento $\mathrm{C}$ dessa mesma marca houve apenas à ausência do lote no rótulo. De acordo com Marins et al. (2008) as informações nutricionais podem auxiliar na escolha de alimentos mais saudáveis, enquanto a análise da lista de ingredientes pode possibilitar a verificação 
da frequência em que ingredientes como o sal, o açúcar e a gordura são utilizados em produtos industrializados.

Os produtos avaliados apresentaram-se de diversas formas durante a coleta dos dados nos estabelecimentos. As linguiças e salsichas eram conservadas e disponibilizadas aos consumidores sempre na forma congelada, os presuntos e salames conservados de forma resfriada e o jerked beef mantido em temperatura ambiente, seguindo assim as recomendações dos estabelecimentos de POA.

Tabela 1- Percentual de conformidades e não conformidades na rotulagem de linguiças de diferentes marcas, comercializadas em três estabelecimentos no município de Bom Jesus-PI

Estabelecimentos

A

B

$\mathrm{C}$

$\begin{array}{ccccccc}\text { Marcas } & \mathrm{C}(\%) & \mathrm{NC}(\%) & \mathrm{C}(\%) & \mathrm{NC}(\%) & \mathrm{C}(\%) & \mathrm{NC}(\%) \\ \text { I } & 100 & 0 & 100 & 0 & 100 & 0 \\ \text { II } & 100 & 0 & 100 & 0 & 100 & 0 \\ \text { III } & 84,37 & 15,62 & 84,37 & 15,62 & 93,87 & 3,12\end{array}$

C - conformidades; NC - não conformidades

Pôde-se observar uma frequência das mesmas marcas sendo comercializadas nos três supermercados para quase todos os produtos, sendo o jerked beef o produto encontrado em menor variedade nos estabelecimentos envolvidos na pesquisa. Todos os produtos eram procedentes de estabelecimentos localizados fora do $\mathrm{Na}$ avaliação dos diferentes rótulos das marcas de salsichas (Tabela 2), verificou-se a ausência de não conformidades para a marca I dentro dos estabelecimentos A, B e C, havendo assim estado do Piauí e possuíam o selo do SIF. Sendo assim, constatou-se que existe uma dependência do abastecimento local de produtos oriundos principalmente da região sul e sudeste do Brasil. Tal fato se deve pela ausência de indústrias certificadas na região para elaboração dos produtos avaliados na pesquisa.

uma maior confiabilidade do produto sabendo que estão dentro das legislações recomendadas. A não conformidade encontrada foi somente para na marca II, sendo a mesma relacionada a ausência do 
lote. Esta informação deveria estar presente nos produtos analisados de acordo com as recomendações da Instrução Normativa $\mathrm{n}^{\mathrm{o}} 22$ do MAPA (BRASIL, 2005).

Martins et al., (2009) ao avaliar rótulos de produtos embutidos de suínos, verificou que as informações referentes ao nome do produto, à data de fabricação e de validade, lista de ingredientes, SIF e nome e endereço dos fabricantes apareceram nos rótulos de todos produtos analisados. Todas as marcas avaliadas apresentaram dados sobre a informação nutricional complementar dos produtos, mas não estavam, em alguns casos, de acordo com a Resolução no 360, de 23 de dezembro de 2003 da ANVISA, pois omitiam algumas informações como a declaração do teor de gordura trans.

Tabela 2- Percentual de conformidades e não conformidades na rotulagem de salsichas de diferentes marcas, comercializadas em três estabelecimentos no município de Bom Jesus-PI

Estabelecimentos

\begin{tabular}{ccccccc}
\hline & \multicolumn{2}{c}{ A } & \multicolumn{2}{c}{ B } & \multicolumn{2}{c}{ C } \\
\hline Marcas & $\mathrm{C}(\%)$ & $\mathrm{NC}(\%)$ & $\mathrm{C}(\%)$ & $\mathrm{NC}(\%)$ & $\mathrm{C}(\%)$ & $\mathrm{NC}(\%)$ \\
& 100 & 0 & 100 & 0 & 100 & 0 \\
\hline I & 96,87 & 3,12 & 96,87 & 3,12 & 96,87 & 3,12 \\
\hline II & & & & & &
\end{tabular}

C - conformidades; NC - não conformidades

Observou-se uma variação na disponibilidade de diferentes marcas dos presuntos nos estabelecimentos A e B. No estabelecimento A foi detectado a presença de apenas uma marca, já no estabelecimento B foram encontras duas variedades, enquanto para o estabelecimento $\mathrm{C}$ as três variedades estavam presentes. Os percentuais de não conformidades para os presuntos foram semelhantes na marca $I$ entre os estabelecimentos A, B e C como mostra a Tabela 3.
Entre as não conformidades presentes no rótulo destes produtos foram constatadas ausência do número do lote nestes rótulos comercializados nos três estabelecimentos. Já para as marcas II e III dos estabelecimentos $\mathrm{A}, \mathrm{B}$ e $\mathrm{C}$ não foram encontradas não conformidades. Moura et al. (2012) também encontrou falhas na rotulagem de presuntos fatiados $\mathrm{e}$ embalados em uma pesquisa realizada na cidade de Uruçuí-PI. O referido autor relata ainda que a fiscalização quanto ao cumprimento da legislação para a 
rotulagem, rotulagem nutricional e rigorosas (MOURA et al., 2012). temperatura de conservação não são

Tabela 3- Percentual de conformidades e não conformidades na rotulagem de presuntos de diferentes marcas, comercializadas em três estabelecimentos no município de Bom Jesus-PI

\section{Estabelecimentos}

\begin{tabular}{ccccccc}
\hline & \multicolumn{2}{c}{ A } & \multicolumn{2}{c}{ B } & \multicolumn{2}{c}{ C } \\
\hline Marcas & $\mathrm{C}(\%)$ & $\mathrm{NC}(\%)$ & $\mathrm{C}(\%)$ & $\mathrm{NC}(\%)$ & $\mathrm{C}(\%)$ & $\mathrm{NC}(\%)$ \\
& & & & & & \\
\hline I & 96,87 & 3,12 & 96,87 & 3,12 & 96,87 & 3,12 \\
II & - & - & 100 & 0 & 100 & 0 \\
\hline III & - & - & - & - & 100 & 0 \\
\hline C - conformidades; NC - não conformidades & & & & & \\
\hline
\end{tabular}

Nos supermercados A e B foi detectada a presença de apenas uma marca de salame. Já no estabelecimento C foram encontradas duas variedades. O percentual de não conformidades da marca I nos três estabelecimentos permaneceu em 3,12\% assim como mostra a Tabela 4.

Nesta marca verificou-se que a informação sobre o teor de gorduras trans não estava presente. Para a marca II não foram encontradas informações incorretas na rotulagem.

Apenas uma marca de jerked beef foi encontrada nos três estabelecimentos.

Não foram detectadas não conformidades na rotulagem destes produtos, bem como sua venda era feita exclusivamente na embalagem original em todos os estabelecimentos. Nos produtos fracionados (linguiça, salsicha, presunto) os valores de não conformidades foram relativamente altos, conforme os dados apresentados na Tabela 5. Dentre as não conformidades verificadas, destaca-se a ausência das seguintes informações: nome e endereço do fabricante, denominação e venda do alimento, lista de ingredientes, conteúdos líquidos, identificação de origem, data de fabricação, prazo de validade e ausência da identificação do lote. 
Tabela 4- Percentual de conformidades e não conformidades na rotulagem de salames de diferentes marcas, comercializadas em três estabelecimentos no município de Bom Jesus-PI

\begin{tabular}{ccccccc}
\hline & \multicolumn{9}{c}{ Estabelecimentos } \\
\hline Marca & $\mathrm{C}(\%)$ & $\mathrm{NC}(\%)$ & $\mathrm{C}(\%)$ & $\mathrm{NC}(\%)$ & $\mathrm{C}(\%)$ & $\mathrm{NC}(\%)$ \\
\hline I & 96,87 & 3,12 & 96,87 & 3,12 & 96,87 & 3,12 \\
\hline II & - & - & - & - & 100 & 0 \\
\hline
\end{tabular}

C - conformidades; NC - não conformidades

Na Tabela 5 mostra o percentual de não conformidades na rotulagem de produtos fracionados, comercializados em três estabelecimentos no município de Bom Jesus-PI, no qual constatou-se também que as embalagens originais não eram higienizadas após fracionamento e o produto entrava em contato permanente com superfícies externas do plástico podendo causar certas doenças ao consumidor. Para os produtos fracionados dos estabelecimentos A e B, apenas o prazo de validade, identificação do lote e data de fracionamento estavam presentes nas embalagens.

Já para o estabelecimento $\mathrm{C}$ todas as não conformidades citadas anteriormente foram constatadas nas embalagens fracionadas, totalizando $100 \%$ de inconformidades nestes produtos fracionados.

Em comparação aos percentuais dos produtos vendidos nas embalagens originais e os fracionados, percebe-se que houve um maior número de não conformidades nos produtos fracionados, tendo em vista que ainda não existe uma legislação oficial que defina regras claras sobre as informações consideradas obrigatórias em produtos fracionados.

Caso semelhante foi relatado por Santos et al. (2015) que encontrou 100\% de falhas em produtos cárneos fracionados, comercializados em Teresina-PI. O autor ainda ressalta que essas falhas detectadas podem inclusive, comprometer a saúde do consumidor, tendo em vista que o mesmo não está tendo acesso à informação.

Tabela 5- Percentual de não conformidades na rotulagem de produtos fracionados, comercializados em três estabelecimentos no município de Bom Jesus-PI.

\begin{tabular}{cccc}
\hline & \multicolumn{3}{c}{ Não Conformidades dos Produtos Fracionados (\%) } \\
\hline Estabelecimentos & \multicolumn{3}{c}{ Produtos } \\
\cline { 2 - 4 } & Linguiça & Salsicha & Presunto \\
\hline A & 66,66 & 66,66 & 66,66 \\
\hline B & 66,66 & 66,66 & 66,66 \\
\hline C & 100 & 100 & 100 \\
\hline
\end{tabular}




\section{Conclusão}

Os supermercados analisados apresentaram pouca variação na distribuição das marcas dos produtos analisados. $\mathrm{O}$ percentual de não conformidades foi maior nos produtos

\section{Referências bibliográficas}

1. ALMEIDA-MURADIAN, L.B.; PENTEADO, M.V.C. Vigilância Sanitária: Tópicos sobre Legislação e Análise de Alimentos. Rio de Janeiro: Guanabara Koogan, 2007. 203p.

2. BRASIL. Ministério da Agricultura, Pecuária e Abastecimento. Secretaria de Defesa Agropecuária. Resolução no 08 de 24 de setembro de 2001. Resolve sobre análise técnica e o registro da rotulagem de produtos de origem animal. D.O.U..1 de outubro de 2001, s. 1, p. 65, 2001.

3. BRASIL. Ministério da Agricultura, Pecuária e Abastecimento. Decreto 9.013, de 29 de março de 2017. Regulamento de inspeção industrial e sanitária de produtos de origem animal. Altera o Regulamento da Inspeção Industrial e Sanitária de Produtos de Origem Animal, aprovado pelo Decreto $\mathrm{n}^{\circ}$ 30.691, de 29 de março de 1952. Brasília, 29 de março de 2017.

4. BRASIL. Ministério da Agricultura e do Abastecimento. Secretaria de Defesa Agropecuária. Instrução Normativa no. 04, de 31 de março de 2000a. Regulamentos técnicos de identidade e qualidade de carne mecanicamente separada, de mortadela, de linguiça e de salsicha. D.O.U.. Alterada pela Instrução Normativa $n^{\circ} 36$, de 13 de outubro de 2011.

5. BRASIL. Ministério da Agricultura e do Abastecimento. Secretaria de Defesa Agropecuária. Instrução Normativa no. 21, de 31 de março de 2000b. Regulamentos técnicos de identidade e qualidade de patê, de bacon ou barriga defumada e de lombo suíno. D.O.U. 03 de agosto de 2000, s. 1, p. 12-15, 2000. fracionados, em comparação aos produtos originais. Os produtos fracionados não exibem todas as informações necessárias à compreensão dos consumidores, impedindo dessa forma, a rastreabilidade do produto.

6. BRASIL. Ministério da Agricultura e do Abastecimento. Secretaria de Defesa Agropecuária. Instrução Normativa no. 22, de 31 de março de 2000c. Regulamentos técnicos de iden-tidade e qualidade de copa, de jerked beef, de presunto tipo parma, de presunto cru, de salame, de salaminho, de salame tipo alemão, de salame tipo calabres, de salame tipo friolano, de salame tipo napolitano, de salame tipo hamburgues, de salame tipo italiano, de salame tipo milano, de linguiça colonial e pepperoni. Alterada pelas Instruções Norma-tivas no. 55, de 7 de julho de 2003 e 43, de 7 de novembro de 2011.

7. BRASIL. Ministério da Agricultura, Pecuária e Abastecimento. Secretaria de Defesa Agropecuária. Instrução Normativa no. 83, de 21 de novembro de 2003. Aprovar regulamentos técnicos de identidade e qualidade de carne bovina em conserva (corned beef) e carne moída de bovino. D.O.U.. 24 de novembro de 2003, 2003.

8. BRASIL. Agência Nacional de Vigilância Sanitária (ANVISA). Resolução RDC No 360, de 23 de dezembro de 2003. D.O.U. de 23/12/2003. Disponível em: http://portal.anvisa.gov.br/documents/33880/2568070/res0360_23_12_2003. $\mathrm{pdf} / 5 \mathrm{~d} 4 \mathrm{fc} 713-9 \mathrm{c} 66-4512-\mathrm{b} 3 \mathrm{c} 1-\mathrm{afee} 57 \mathrm{e} 7 \mathrm{~d} 9 \mathrm{bc}$. Acesso em: 19 abril de 2018.

9. BRASIL. Ministério da Agricultura, Pecuária e Abastecimento. Instrução Normativa $n^{0} 22$, de 24 de novembro de 2005. Disponível em:http://ambbrasilia.esteri.it/resource/2009/09 /INTo-tale.pdf. Acesso em: 02 fev. 2018.

10. CODEX ALIMENTARIUS. Codex Stan 1, 1985. Norma general para el etiquetado de los alimentos preenvasados, 2010. Disponível em: http://www.fao.org/input/download/stand- 
ards/32/cxs_001s.pdf. Acesso em: 12 jan. 2018.

11. GONÇALVES, C. Alimento seguro é um direito fundamental, 2009. Sistema de gestão da qualidade em alimentos. Disponível em: http://clovisgoncalves.blogspot.com.br/2009/1 0/alimento-seguro-e-um-direito.html. Acesso em: 02 abr. 2018.

12. MACHADO, R.L.P. Manual de rotulagem de alimentos. $1^{\circ}$ edição. Rio de Janeiro - RJ. Embrapa Agroindústria de Alimentos, 2015. $26 \mathrm{p}$.

13. MARINS, R.B.; COUTO, S.C.; PERES, F. Avaliação qualitativa do hábito de leitura e entendi-mento: recepção das informações de produtos alimentícios. Ciência Tecnologia Alimentos, Campinas, v.28, n.3, p.579-585, 2008.

14. MARTINS, T.D.D; BEZERRA, W.I; MOREIRA, R,T; SILVA, L.P.G.; BATISTA, E.S. Mercado de embutidos de suínos: comercialização, rotulagem e caracterização do consumidor. Revista Brasileira de Saúde Produção Animal, v.10, n.1, p.12-23, jan/mar, 2009.

15. MOURA, R.L.; SANTOS, J.M.S.; GUIMARÃES, F.R.; FREITAS, R.F.; Avaliação da rotulagem de presuntos fatiados comercializados na cidade de Uruçuí-PI. In: VII CONNEPI- Congresso Norte Nordeste de Pesquisa e Inovação. 2012.

16. SANTOS, T.P; PEREIRA, R.E.E; JÚNIOR, M.H.K. Análise da rotulagem de produtos cárneos comercializados em Teresina, Piaú. Revista Brasileira de Higiene e Sanidade Animal, v.9, n.3, p. 364-379, 2015. http://dx.doi.org/ 10.5935/19812965.20150034 